\title{
Completeness of experimental fission product yields in EXFOR database
}

\author{
Takanari Fukuda ${ }^{1,2, *}$, Shin Okumura ${ }^{2, \dagger}$, and Naohiko Otuka ${ }^{2, \ddagger}$ \\ ${ }^{1}$ Cooperative Major in Nuclear Energy, Graduate School of Advanced Science and Engineering, Waseda University, Tokyo 169-8555, \\ Japan \\ ${ }^{2}$ Nuclear Data Section, Division of Physical and Chemical Sciences, Department of Nuclear Sciences and Applications, International \\ Atomic Energy Agency, A-1400 Wien, Austria
}

\begin{abstract}
The Nuclear Reaction Data Centres (NRDC) collaborate on worldwide compilation and dissemination of experimental nuclear reaction data by developing and maintaining the EXFOR database. With the growing interests in the fission product yields of various projectiles and a wide range of energies, several evaluation activities are ongoing. In line with these activities, the IAEA conducted completeness check of fission product yield data in EXFOR against two experimental datasets developed by the ENDF and UKFY library evaluators. The present status and statistics of these datasets as well as the result of the EXFOR completeness review are presented.
\end{abstract}

\section{Introduction}

The fission product yield (FPY) is an important ingredient of nuclear data in both fundamental physics and nuclear applications. The FPY plays a key role to understand the fission process and is also responsible for the abundance of elements and nuclides synthesised in the $r$ process. In nuclear applications, the FPY has been used in calculations of reactor criticality, burn-up, decay heat, reactor design, fuel handling, inventory and safety. The IAEA Nuclear Data Section (NDS) has extensively reviewed the user needs in these application fields in the previous IAEA Coordinated Research Projects (CRPs), e.g., "Compilation and evaluation of fission yield nuclear data" (1991-1996) [1] and "Fission Product Yield Data for the Transmutation of Minor Actinide Nuclear Waste" (19972002) [2]. Also the Technical Meeting on "Fission Product Yields: current status and perspectives" (2016) [3] addressed the importance of the experimental and the evaluated FPY data dissemination.

The experimental FPY has always been the foundation of the FPY data evaluations, where a complete set of the experimental FPY data is crucial. The NDS has been serving an experimental nuclear reaction database (EXFOR) [4] under long-term cooperation with other Nuclear Reaction Data Centres (NRDC) [5]. The EXFOR database contains a wide range of physical quantities of nuclear reactions including FPY. The EXFOR coding rules of the FPY and the other fission quantities were established in 1970s. Since then, the NRDC network has devoted an effort to accumulate FPY data constantly in EXFOR. In parallel with this, the pioneers in this field built their

\footnotetext{
*e-mail: sun-village@akane.waseda.jp

†e-mail: s.okumura@iaea.org

¥e-mail: n.otsuka@iaea.org
}

own compilations to produce evaluated FPY libraries, e.g. Meek and Rider [6] and Crouch [7]. In order to crosscheck EXFOR with such evaluator's experimental dataset, several completeness checks has taken place in the past $[8,9]$. However, such crosschecking has not been performed in the last two decades.

The recent increase in demand of the FPY data in various fields has triggered vigorous activity of developing new methods, codes and models for FPY evaluation. However, the current status of such developments has not yet reached at the level of producing the accurate FPY data without support of the experimental data. It is widely recognised that the experimental FPY dataset is important as a common basis of new evaluation [10]. The NDS plans to launch a new CRP in 2020 to update the evaluated FPY data for the neutron-induced fission of some important actinides. Under the circumstance, we conducted a completeness check of FPY data in EXFOR by comparison with the references cited in the summary reports of the ENDF-B/VI [11] and UKFY3.0 [12] evaluations.

In this paper, we report the current status of our assessment including the number of relevant FPY data identified for additional EXFOR compilation as well as statistics of projectiles and target nuclides in the ENDF-B/VI and UKFY3.0 evaluation summaries.

\section{Completeness assessment}

\subsection{Scope of our assessment}

In the nuclear fission, the fissioning nucleus separates into two primary fission fragments that rapidly de-excite through the prompt gamma and neutron emissions to reach either the ground or a metastable state. The secondary (post-neutron emission) fission fragments, also refereed 
Table 1. Typical fission fragment and product yield quantities defined in the EXFOR dictionary. Each comma-separated field corresponds to Product (SF4), Branch (SF5), and Parameter (SF6) in the REACTION code in EXFOR*. See EXFOR Compiler's Manual (LEXFOR) [13] for more detailed descriptions of the coding rules.

\begin{tabular}{llll}
\hline Stage & Y(Z,A,M) & Y(A) & Y(Z) \\
\hline Primary fission fragment & ELEM/MASS,TER,FY & MASS,PRE,FY & ELEM,PRE,FY \\
& 2-HE-4,TER,FY & MASS,PRV,FY & ELEM,TER,FY \\
\hline \multirow{2}{*}{ Secondary fission fragment } & ELEM/MASS,IND,FY & \multirow{2}{*}{ MASS,SEC,FY } & ELEM,CHG,FY \\
(Independent fission product) & 55-CS-136,IND,FY & & \\
& ELEM/MASS,IND,FY,FRC & & \\
& 0-G-0,PR,FY & & ELEM,CUM,FY \\
Cumulative fission product & ELEM/MASS,CUM,FY & \multirow{2}{*}{ MASS,CHN,FY } & \\
& 42-MO-99,CUM,FY & & \\
& ELEM/MASS,CUM,FY,FRC & & \\
\hline
\end{tabular}

${ }^{*} \mathrm{CHG}=$ charge, $\mathrm{CHN}=$ chain, $\mathrm{CUM}=$ cumulative, $\mathrm{FRC}=$ fractional, $\mathrm{FY}=$ fission yield, $\mathrm{TER}=$ ternary, IND = independent, $\mathrm{PR}=$ prompt, $\mathrm{PRE}=$ primary, $\mathrm{PRV}=$ provisional, $\mathrm{SEC}=$ secondary .

as independent fission products, are usually neutron rich and undergo $\beta$ decay. The final products after $\beta$ decay are called cumulative fission products. Corresponding to these three stages in the nuclear fission process, the EXFOR dictionary defines quantity codes to distinguish them each other. Typical quantity codes are summarised in Table 1 .

When a fission fragment/product is specified by a combination of charge $Z$, mass $A$ and isomeric state $M$, its FPY $Y$ is a function of $Z, A$ and $M$, i.e., $Y(Z, A, M)$. Sometimes FPY is specified only by its mass or charge, i.e., mass distribution $Y(A)$ or charge distribution $Y(Z)$. The most probable mass $A_{p}$, which is the mean mass within the distribution of the element or the mass distribution $Y(A)$ of light or heavy fragments, is also considered as a FPY quantity. Similar to $A_{p}$, the mean charge is called most probable charge $Z_{p}$ which is also a FPY quantity.

The current evaluated nuclear data libraries incorporate the independent and cumulative FPYs only because the primary fission fragments decay very rapidly than the experimental time scale. By closely following this reason, evaluation summaries of ENDF-B/VI [11] and UKFY3.0 [12] are composed of the quantities of after prompt particle emissions for neutron-induced and spontaneous fission. In the next section, the procedure of our completeness check against these ENDF-B/VI and UKFY3.0 compilations is described.

\subsection{Procedure of the completeness assessment}

\subsubsection{Preparation of reference lists}

The detail of the assessment procedure is described in Ref. [14] and Ref. [15]. A brief description will be given here. We extracted bibliographies from the citation lists of the ENDF-B/VI and UKFY3.0 evaluation summaries, and converted them to text format. The EXFOR reference code (e.g., J , PR, 60, 534, 1941 for Phys. Rev. 60(1941)534) was generated according to the EXFOR dictionary by an Excel VBA code. The generated EXFOR reference code was double-checked manually followed by a brief check using Python script. When the reference was not compiled in EXFOR nor in CINDA, we checked the correctness of its bibliography (e.g., volume, page, publication year) by various means. For journal articles, Crossref database was used. Otherwise we tried to identify them via online search engines or electronic repositories such as Google Scholar and the International Nuclear Information System (INIS). If such attempt did not succeed, we tried to obtain hard copies from libraries of universities or research institutes through the IAEA Library. We also collected a copy of each article missing in EXFOR, and checked its relevance to EXFOR compilation. The references extracted from the ENDF-B/VI and UKFY3.0 evaluation summaries were merged, stored into the SQL database, and sorted by the author and EXFOR reference code.

The ENDF/B-VI evaluation summary cites 1,602 references, of which 924 references provide FPY data adopted by the evaluators and were for our present assessment, while the rest 678 references are reserved for future analysis. The UKFY3.0 evaluation summary cites 646 references, and all of them were for our present assessment. It was revealed that about 600 references are cited in both ENDF/B-VI and UKFY3.0 evaluation summaries, about 1,200 references are unique, and the duplication was eliminated from the merged list.

\subsubsection{Check against EXFOR and CINDA}

The status of each reference in the EXFOR [4] and CINDA [16] databases was checked with the EXFOR reference code, first author, projectile and target nuclide as keys. We categorised each article to one of the following statuses:

Exist: If the reference was in an EXFOR entry and all target nuclides in the CINDA records of the same reference are seen in the REACTION codes of the EXFOR entry, the reference was categorised as "Exist". The reference requires no further action.

New Entry: If the reference was not in EXFOR and the reference reports FPY data discussed in Sec. 2.1, the reference was categorised as "New Entry". The reference is for intensive FPY EXFOR compilation by NRDC.

Not FPY: If the reference was not in EXFOR and the reference reports EXFOR related fission data other than those discussed in Sec. 2.1, the reference was categorised as 
"Not FPY". The reference is for regular retroactive EXFOR compilation by NRDC.

Check: If the reference was not in EXFOR but CINDA indicates the same experimental work is compiled in EXFOR from another reference (i.e., two references are in the same "block" in CINDA), the reference was categorised as "Check". This situation may happen when a data set published in a reference was compiled in EXFOR and then the data set was superseded by another reference which NRDC was not aware. Even if all data in the reference have been already compiled, the reference must be added to the EXFOR entry as an additional reference. This is because it will be an evidence that NRDC has already checked the reference, and also EXFOR users will find the FPY data set by using the reference as a key. When we found a reference of this category, the reference in EXFOR from the same experimental work was recorded in our list.

Not Relevant: If the reference is not relevant to EXFOR (e.g., evaluation, calculation, review), the reference was categorised as "Not Relevant". The reference requires no further action.

Not Found: If the access to the reference was not realistic (e.g., private communications, classified reports, Ph.D. theses) or its bibliographic information was incomplete for librarians, it was categorised as "Not Found".

\section{Assessment results}

\subsection{Statistics of projectiles and target nuclides}

The statistics of the projectiles and target nuclides were analysed for the references cited in the ENDF/B-VI and UKFY3.0 evaluation summaries and also compiled in the CINDA [16] which includes bibliographies imported from the EXFOR [4]. Both ENDF/B-VI and UKFY3.0 compilations comprise approximately 25\%, 10\%, 9\%, $8 \%$ and $5 \%$ FPY from the neutron-induced fission of ${ }^{235} \mathrm{U},{ }^{239} \mathrm{Pu}$, ${ }^{238} \mathrm{U},{ }^{233} \mathrm{U}$, and ${ }^{232} \mathrm{Th}$, respectively (Fig. 1). We found that about $70 \%$ and $8 \%$ of the references are from neutroninduced and spontaneous fission, respectively (Fig. 2). There are a few references reporting $(\gamma, \mathrm{f}),(\mathrm{d}, \mathrm{f}),(\alpha, \mathrm{f})$, and $(\mathrm{p}, \mathrm{f})$ FPY.

\subsection{The EXFOR relevant references}

Table 2 shows the number of references categorised during our completeness check. The number of references that need to be compiled ("New Entry") are 194 after exclusion of the overlapped references between two sources, while the references that need to be checked with existing EXFOR entries ("Check") are 54.

In parallel with our assessment, the US National Nuclear Data Center (NNDC) also performed a similar assessment [17] by extracting FPY related references in the NSR database [18] and not in EXFOR. We converted their list to our text format, and stored the bibliographies in the SQL database for further analysis. The NNDC assessment covers not only FPY but also other fission quantities such as total kinetic energies of fission fragments

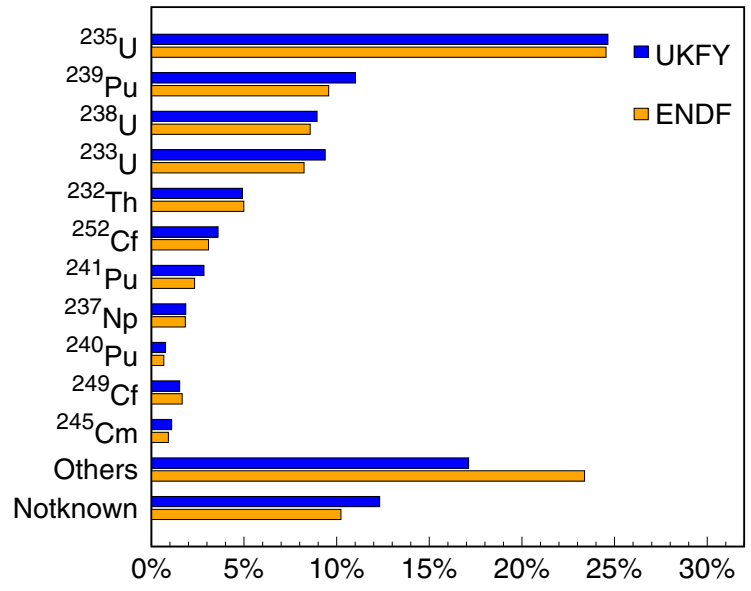

Figure 1. Target nuclides cited in the evaluation summaries of ENDF/B-VI [11] and UKFY3.0 [12]. Orange and blue bars represent percentages of target nuclides in Ref. [11] and Ref. [12], respectively. "Others" is the total of other actinides. For references with no nuclide or reaction information in CINDA are categorised as "Notknown".

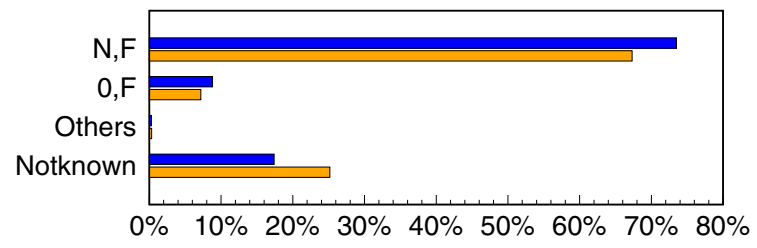

Figure 2. Reaction categories, (N,F): neutron-induced and (0,F): spontaneous fission. See legend to Fig. 1.

and prompt neutron spectra. Only 33 references are overlapped between NDS and NNDC lists, and they are mainly for neutron-induced FPY.

During this work, we also identified the institute where the experiment took place. Each reference in the NDS and NNDC lists was assigned to the responsible centre according to the location of the institute (e.g., data measured in China are for compilation by China Nuclear Data Centre), and the NRDC 2019 meeting [19] officially requested the responsible data centres to compile the FPY data published in the references identified by two assessments. The references assigned to the data centres are listed in Ref. [15] for our assessment and Ref. [20] for the NNDC assessment. The list of the "Exist", "New Entry" and "Check" references from our assessment is also appended to Ref. [10].

\section{Conclusion and outlook}

We reviewed the completeness of the FPY data in the EXFOR database by crosschecking against the citation lists of the ENDF-B/VI and UKFY3.0 evaluation summaries. It was found that approximately 194 references are relevant to new compilation of EXFOR entry ("New Entry"), and about 54 references must be checked with the existing EXFOR entries ("Check") to ensure that the all experimental FPY data are properly compiled. The compilation has been started by data centres. 
Table 2. The number of FPY references categorised during our assessment for those cited in evaluation summaries of ENDF/B-VI [11] and UKFY3.0 [12]. See the main text for the definition of each category.

\begin{tabular}{lccccc}
\hline Source & Exist & New $^{*}$ & Check & Not $^{\dagger}$ & Total \\
\hline ENDF/B-VI & 618 & 154 & 36 & 116 & 924 \\
UKFY3.0 & 409 & 139 & 32 & 64 & 645 \\
Total $^{\ddagger}$ & 720 & 194 & 54 & 167 & 1203 \\
\hline
\end{tabular}

$*$ New $=$ New Entry.

$\dagger$ Not $=$ Not FPY+Not Relevant+Not Found.

$\star$ Total $=$ Total after elimination of overlap.

Apart from these FPY references, we found (1) about 167 references reporting fission quantities other than FPY ("Not FPY") and (2) 678 references cited in the ENDF/BVI evaluation summary but not quoted in its experimental database. We will continue a similar assessment for these remaining references.

In the mean time, we found some EXFOR entries that do not follow the current EXFOR coding rule (e.g., the total kinetic energy tagged with , AKE, FF which must be , AKE , LF+HF), and we plan to assess and fix the problems to make EXFOR retrieval much easier.

\section{Acknowledgement}

We would like to express our thanks to Toshihiko Kawano (LANL Theoretical Division) for fruitful discussion and Lidija Vrapcenjak (IAEA Nuclear Data Section) for collection of article copies. T.F. acknowledges support from the Strategic Nuclear Education Program entrusted to the Japanese University Network for Global Nuclear Human Resource Development (JUNET-GNHRD) by the Ministry of Education, Culture, Sports, Science and Technology of Japan (MEXT).

\section{References}

[1] International Atomic Energy Agency, Tech. Rep. IAEA-TECDOC-1168, International Atomic Energy Agency (2000)

[2] International Atomic Energy Agency, Tech. Rep. STI/PUB/1286, International Atomic Energy Agency (2008)
[3] P. Dimitriou, F.J. Hambsch, S. Pomp (eds.), Tech. Rep. INDC(NDS)-0713, International Atomic Energy Agency (2016)

[4] Experimental Nuclear Reaction Data (EXFOR), https://www-nds.iaea.org/exfor/

[5] N. Otuka, E. Dupont, V. Semkova, B. Pritychenko, A. Blokhin, M. Aikawa, S. Babykina, M. Bossant, G. Chen, D. Dunaeva et al., Nucl. Data Sheets 120, 272 (2014)

[6] M. Meek, B. Rider, Tech. Rep. NEDO-12154-1, General Electric Co., Pleasanton, Calif. (USA). Vallecitos Nuclear Center (1974)

[7] E. Crouch, Atom. Data Nucl. Data Tables 19, 417 (1977)

[8] P. Johnston, NRDC Memo 4C-2/115 (1980)

[9] M. Lammer (ed.), Tech. Rep. INDC(NDS)-261, International Atomic Energy Agency (1991)

[10] M. Fleming, T. Kawano, N. Otuka (eds.), Tech. Rep. INDC(NDS)-793, International Atomic Energy Agency (2019)

[11] T.R. England, B. Rider, Tech. Rep. ENDF-349, LA-UR-94-3106, Los Alamos National Laboratory (1994)

[12] R. Mills, Ph.D. thesis, University of Birmingham (1995)

[13] O. Schwerer (ed.), Tech. Rep. IAEA-NDS-208 Rev.2015/08, International Atomic Energy Agency (2015)

[14] S. Okumura (2019), nRDC Meeting 2019

[15] S. Okumura, NRDC Memo CP-D/979 (2019)

[16] Computer Index of Nuclear Reaction Data (CINDA), https://www-nds.iaea.org/cinda/

[17] P. Pritychenko, O. Schwerer, J. Totans, V. Zerkin, in these proceedings (to be published)

[18] B. Pritychenko, E. Běták, M. Kellett, B. Singh, J. Totans, Nucl. Instrum. Meth. A 640, 213 (2011)

[19] M. Fleming, N. Otuka (eds.), Tech. Rep. INDC(NDS)-72, International Atomic Energy Agency (2019)

[20] O. Schwerer, P. Pritychenko, NRDC Memo CPC/464, 465 and 466 (2019) 